\title{
Profil épidémiologique des maladies cardiovasculaires dans la Ville de Meknès (Maroc)
}

\author{
Ibrahim El Ghazi, PhD \\ Imane Berni, PhD \\ Université Moulay Ismail, Cluster of Competence \\ "Environment \& Health", Meknes, Morocco \\ Aziza Menouni, PhD \\ Université Moulay Ismail, Cluster of Competence \\ "Environment \& Health", Meknes, Morocco \\ Centre of Environment and Health, KU Leuven, Leuven Belgium \\ Prof. Marie-Paule Kestemont, \\ Université Catholique de Louvain, Louvain la neuve, Belgium \\ Prof. Mohammed Amane, \\ Prof. Samir El Jaafari, \\ Université Moulay Ismail, Cluster of Competence \\ "Environment \& Health", Meknes, Morocco
}

Doi: 10.19044/esj.2018.v14n33p49 URL:http://dx.doi.org/10.19044/esj.2018.v14n33p49

\begin{abstract}
Introduction: In Morocco, very little published work has focused on cardiovascular diseases. As a result, the main objective of this research is to evaluate the epidemiological profile of cardiovascular diseases in patients who are hospitalized and treated in the cardiology department of Mohammed V Hospital, Meknes (Morocco). Methods: This is a retrospective descriptive and analytical study of 1112 patients admitted to this department over a fouryear period (from January 1, 2011 to December 31, 2014). Results: $54.49 \%$ of patients were women and the most affected age group was 45-64 years old $(42.08 \%)(\mathrm{p}<0.001)$. The most common pathologies among hospitalized patients were: ischemic heart disease which represented the first cause of hospitalization with 341 cases corresponding to $30.66 \%$, followed by heart failure $(20.59 \%)$. Ischemic heart disease was more common in men $(p=0.05)$ while women were more affected by high blood pressure $(\mathrm{p}=0.0096)$, heart failure $(\mathrm{p}=0.06)$ and venous thrombosis $(\mathrm{p}<0.05)$. Conclusion: Cardiovascular pathologies represent a real health and socio-economic problem for families and health facilities. The frequency and severity of these
\end{abstract}


diseases should prompt us to do further research on this topic to find the most plausible solutions to reduce negative impacts.

Keywords: Cardiovascular diseases, ischemic heart disease, heart failure, Meknes

\section{Resume}

Introduction : Au Maroc très peu de travaux publiés se sont intéressés de façon rigoureuse aux maladies cardiovasculaires. De ce fait, l'objectif majeur de cette recherche est d'évaluer le profil épidémiologique des maladies cardiovasculaires des sujets hospitalisés et traités au service de cardiologie de l'Hôpital Mohammed V de Meknès (Maroc). Méthodes: Il s'agit d'une étude rétrospective descriptive et analytique portant sur 1112 patients admis au service de cardiologie de l'Hôpital Mohammed V, sur une période de quatre ans (du 1er janvier 2011 au 31 décembre 2014). Résultats : cinquante-quatre pour cent des patients étaient des femmes et la tranche d'âge la plus touchée était celle de 45-64 ans $(42,08 \%)(\mathrm{p}<0,001)$. Les pathologies les plus répandues parmi les sujets hospitalisés : la cardiopathie ischémique qui a représenté la première cause d'hospitalisation avec 341 cas correspondant à 30,66\%, suivie de l'insuffisance cardiaque $(20,59 \%)$. La cardiopathie ischémique était plus fréquente chez les hommes $(\mathrm{p}=0,05)$ alors que les femmes étaient plus touchées par l'hypertension artérielle ( $p=$ $0,0096)$, l'insuffisance cardiaque $(\mathrm{p}=0,06)$ et la thrombose veineuse ( $\mathrm{p}<$ 0,05). Conclusion : Les pathologies cardiovasculaires représentent un véritable problème à la fois sanitaire et socio-économique pour les familles et les structures sanitaires. La fréquence et la gravité de ces maladies doivent nous inciter à faire des recherches plus poussées à propos de ce sujet afin de trouver les solutions les plus plausibles pour réduire les répercussions négatives.

Mots clés: Maladies cardiovasculaires, cardiopathies ischémiques insuffisance cardiaque, Meknès

\section{Introduction}

Les maladies cardiovasculaires (MCV) constituent l'une des causes majeures de morbidité et de mortalité à l'échelle planétaire (Damorou et al., 2014 ; Gaziano et al., 2011). D'après l'organisation mondiale de la santé (OMS), plus de 17,3 millions de personnes meurent chaque année de MCV, soit $31 \%$ de la mortalité mondiale totale (Damorou et al., 2014). Si en occident la prévalence des MCV est connue, elle n'est pas encore d'actualité dans les pays en voie de développement notamment en Afrique (Kheyi et al., 2016). 
De ce fait, l'objectif majeur de cette recherche est d'évaluer le profil épidémiologique des MCV des sujets ayant séjourné au service de cardiologie de Meknès (Maroc).

\section{Matériels et Méthodes}

Il s'agit d'une étude rétrospective, descriptive et analytique portant sur des patients admis au service de cardiologie de l'Hôpital Mohammed V (Meknès), sur une période de quatre ans allant du 1er janvier 2011 au 31 décembre 2014. Ont été inclus dans ce travail, les sujets résidants à Meknès et ayant été hospitalisés au service de cardiologie de l'hôpital Mohammed V. Les données ont été collectées à partir des registres médicaux d'hospitalisation au service de cardiologie (numéro d'entrée du patient, date d'admission, âge, sexe, diagnostic d'entrée et diagnostic de sortie, adresse). Les données recueillies ont été saisies et analysées par Microsoft Excel 2010 et les calculs des probabilités ont été réalisés par le logiciel R. Le test de Khi2 a été utilisé pour comparer les variables qualitatives. Le seuil de signification retenu était la probabilité $\mathrm{p} \leq 0,05$.

\section{Résultats}

Dans cette étude qui a concerné 1112 patients souffrant de problèmes cardiovasculaires, l'âge moyen des sujets hospitalisés était de 60,59 $\pm 15,31$ ans avec un minimum de 11 ans et un maximum de 105 ans. La médiane d'âge était de 62 ans. Six cent six cas $(54,49 \%)$ sont des femmes et $506(45,50 \%)$ des hommes (Tableau 1). Le sex-ratio était de 1,19 en faveur du sexe féminin. Les patients ayant 50 ans et plus ont représenté 78,68 \% (875 cas), contre 21,32 $\%$ (237 cas) pour les moins de 50 ans (Tableau 1).

\begin{tabular}{|l|l|}
\hline \multicolumn{2}{|l|}{ Tableau 1. Caractéristiques des sujets hospitalisés au service de cardiologie } \\
\hline Caractéristiques & Effectifs $(\mathrm{n}=1112)(\%)$ \\
\hline Sexe & \\
\hline Masculin & $506(45,50)$ \\
\hline Féminin & $606(54,49)$ \\
\hline Âge & \\
\hline Inférieur à 14 ans & $4(0,35)$ \\
\hline Entre 15 et 49 ans & $233(20,95)$ \\
\hline 50 ans et plus & $875(78,68)$ \\
\hline Provenance & \\
\hline Meknes & $947(85,16)$ \\
\hline Ouislane & $143(12,85)$ \\
\hline Toulal & $22(1,97)$ \\
\hline
\end{tabular}


La tranche d'âge la plus touchée était celle de 45-64 ans avec 42,08\% (468 cas) alors que la moins affectée était celle de 0 à 19 ans (8 cas soit 0,46 $\%)(\mathrm{p}<0,001)$. Quatre vingt pour cent $(947$ cas) provenaient de la commune de Meknès, 12,85 \% (143 cas) d'Ouislane et 1,97 \% (22 cas) de Toulal. La cardiopathie ischémique a représenté la première cause d'hospitalisation avec 341 cas correspondant à $30,66 \%$, suivie de l'insuffisance cardiaque $(20,59$ $\%)$.

La cardiopathie ischémique était plus fréquente chez les hommes ( $\mathrm{p}=$ $0,05)$ alors que les femmes étaient plus touchées par l'hypertension artérielle $(\mathrm{p}=0,0096)$, l'insuffisance cardiaque $(\mathrm{p}=0,06)$ et la thrombose veineuse ( $\mathrm{p}$ $<0,05)$.

\section{Discussions}

La cardiopathie ischémique $(\mathrm{CI})$ a représenté le premier motif d'hospitalisation avec 341 cas (soit 30,66 \%). Les hommes ont été légèrement plus affectés que les femmes avec 196 cas $(57,47 \%)$ contre 145 cas $(42,52 \%)$ $(\mathrm{p}=0,005)$. Le sex-ratio était de 1,35 . L'âge moyen des patients était de 62,83 \pm 10 ans (âge extrême 32 - 90 ans). La médiane d'âge était de 63 ans. Cinquante pour cent des cas hospitalisés appartenaient à la tranche d'âge de 45-64 ans alors que seulement 6,45\% avaient moins de 44 ans (p < 0,001). Au Congo Brazzaville, La fréquence de la CI était de 10,7 \%. L’âge moyen était de $66,33 \pm 11,8$ ans, 50,4 \% des patients étaient de sexe féminin et 94,7\% des cas avaient 50 ans ou plus (Ondze-Kafata et al., 2014). Dans notre étude 87,68 $\%$ des cas d'hospitalisation (119 cas) avaient 50 ans ou plus. L'infarctus du myocarde (syndrome coronarien aigu $\mathrm{ST}^{+}$) a été plus fréquente chez les hommes avec $63,97 \%$ contre $36,02 \%$ pour les femmes $(\mathrm{p}=0,0011)$ et le sexratio est de 1,77 . L'âge moyen des patients était de 60,14 $\pm 12,52$ ans (âge extrême 32 - 89 ans). La médiane d'âge était de 60 ans. Cinquante-sept pour cent des cas hospitalisés appartenaient à la tranche d'âge de 45-64 ans alors que seulement 5,60\% avaient moins de 44 ans ( $\mathrm{p}<0,001)$. Une étude réalisée en France a montré que les hommes étaient deux fois plus touchés par l'IDM que les femmes (66,4 \% versus 33,6 \%), l'âge moyen des patients était de 68,2 ans et la proportion des patients de moins de 65 ans était égale à 40,5\%, celle de 65-84 ans à 44,5\% et celle des patients plus âgés à $15 \%$ (De Peretti et al., 2012). Pour le syndrome coronarien aigu ST${ }^{-}$, les hommes ont été plus touchés que les femmes avec 55 cas versus 24 cas $(\mathrm{p}<0,001)$. Le sex-ratio était de 2,29. L'âge moyen a été de $61,35 \pm 10,51$ ans (âge extrême $38-87$ ans). La médiane d'âge a été de 62 ans. Quatre-vingt-dix pour cent des patients avaient 50 ans ou plus $(\mathrm{p}<0,001)$, la tranche d'âge la plus touchée était celle de 4564 ans avec 59,49\% (p < 0,001). A Oujda $54 \%$ des patients hospitalisés pour SCA ST- étaient des hommes, l'âge moyen était de 65 ans (âge extrême 37 - 
90 ans), la tranche d'âge la plus touchée était celle de 65-75 ans et plus de la moitié des cas avaient plus de 65 ans (Aziouaz, 2014).

L'insuffisance cardiaque (IC) représentait le second motif d'hospitalisation avec $20,59 \%$ et a été plus fréquente chez les femmes $(\mathrm{p}<$ $0,05)$ avec un sex-ratio de 1,43. L'âge moyen des patients était de 59,23 \pm 15,98 ans, avec un minimum de 11 ans et un maximum de 96 ans. La médiane d'âge était de 60 ans. Au Togo, les femmes ont été plus touchées que les hommes, l'incidence hospitalière de l'IC était de $25,6 \%$ et l'âge moyen des patients était de 52,2 $\pm 16,7$ ans (Pio et al., 2014). Une étude menée au niveau de l'Hôpital Militaire Mohammed V de Rabat, entre décembre 2008 et 2014, portant sur 424 patients hospitalisés pour IC a montré une prédominance du sexe masculin avec $72 \%$ des cas hospitalisés avec un sex-ratio de 2,5 et l'âge moyen des patients était de 60,91 $\pm 12,77$ ans (Kheyi et al., 2016). Dans notre étude, La tranche d'âge de 45 à 64 ans a été la plus représentée $(43,75 \%)$ et celle de 0 à 19 ans était peu affectée $(1,31 \%)(\mathrm{p}<0,001)$ et les patients ayant 50 ans ou plus ont représenté 76,85\% des sujets hospitalisés pour IC (p < 0,001). Au Niger, la tranche d'âge la plus touchée était celle de 65 ans et plus (36 cas soit 34,96\%) avec une prédominance du sexe masculin. Cinquantesept pour cent (59 cas) des sujets hospitalisés étaient des hommes et $42,70 \%$ (44 cas) des femmes, le sex-ratio a été de 1,34 et l'âge moyen des patients était de 55,05 ans (extrêmes 17 à 96 ans) (Maliki Abdoulaye et al., 2018).

Six pour cent a été le pourcentage des hospitalisations pour thrombose veineuse (TV) et les femmes ont été plus affectées que les hommes $(62,68 \%)$ contre $(37,31 \%)(\mathrm{p}<0,05)$ avec un sex-ratio 1,68. Un résultat similaire a été observé au service de cardiologie de l'hôpital général de grand Yoff de Dakar (Dioum et al., 2017). L'âge moyen de nos patients était de 54,93 $\pm 19,15$ ans (âge extrême $21-90$ ans). Les patients ayant 50 ans ou plus représentaient $55,22 \%$ et la tranche d'âge la plus affectée était celle de 20-44 ans avec 40,29 $\%$. Au Sénégal, l'incidence hospitalière des TV était de 2,78 \%, l'âge moyen était de 51 ans $\pm 19,22$, la tanche d'âge la plus touchée était celle de 40-49 ans et les patients ayant 60 ans ou plus représentaient 36,5\% (Dioum et al., 2017).

Sur les 1112 patients hospitalisés et traités au service de cardiologie, 26 cas ont été admis pour Accidents Vasculaires Cérébraux (AVC) soit une incidence de 2,33\%. Les femmes représentaient 76,92 \% (20 cas) avec un sexratio $(\mathrm{F} / \mathrm{H})$ de 3,33 . La prédominance du sexe féminin a été, également, constatée en Côte d'Ivoire (Kouakou et al., 2015) et au Togo. L'âge moyen de nos patients était de $61 \pm 15,85$ ans (âge extrême $25-81$ ans). Ce résultat a été proche de celui enregistré en Côte d'Ivoire (60 ans) (Kouakou et al., 2015) et au Togo (58,2 ans) (Machihude et al., 2015). Les tranches d'âge les plus touchées étaient celles de 45-64 ans et 65-74 ans et les patients ayant 45 ans et plus représentaient $76,92 \%$ des cas hospitalisés pour AVC. Alors qu'au 
Togo, les tranches d'âge de 40 à 49 ans et de 50 à 59 ans ont été les plus affectées (Machihude et al., 2015). Les AVC représentent un véritable problème à la fois sanitaire et socio-économique puisqu'ils affectent des personnes actives qui vont se transformer après l'incidence de l'AVC en handicapés constituant ainsi un fardeau pour les familles et les structures sanitaires (Machihude et al., 2015).

\section{Conclusion}

Les pathologies cardiovasculaires représentent un véritable problème à la fois sanitaire et socio-économique pour les familles et les structures sanitaires. La fréquence et la gravité de ces maladies doivent nous inciter à faire des recherches plus poussées à propos de ce sujet afin de trouver les solutions les plus plausibles pour réduire les répercussions négatives.

\section{References:}

1. Gaziano TA, Gaziano JM. Braunwald s heart disease: A textbook of cardiovascular medicine. Boston: Elsevier Saunders; 2011.

2. Damorou F, Baragou S, Pio M, et al. (2014). Morbidité et mortalité hospitalière des maladies cardiovasculaires en milieu tropical: exemple d'un centre hospitalier à Lomé (Togo). The Pan African Medical Journal, 17:62.

3. De Peretti C, Chin F, Tuppin P, Danchin D, et al. (2012). Personnes hospitalisées pour infarctus du myocarde en France : tendances 20022008. Bull Epidemiol Hebd, 41:459-65.

4. Ondze-Kafata LI, Bianza JR, Ngolo-Letomo K, Otiobanda GF, Amounya-Zobo S, Bakekolo RP, Gankama T, Gombet T, KimballyKaky G, Nkoua JL. (2014). La cardiopathie ischémique au centre hospitalier et universitaire de Brazzaville : étude de 133 cas. Dakar Med, 59(3) ,150-157.

5. Kheyi J., Benelmakki1 A, Bouzelmat H, Chaib A. (2016). Epidémiologie et prise en charge de l'insuffisance cardiaque dans un centre marocain. Pan African Medical Journal, 24:85.

6. Pio M., Afassinou Y., Pessinaba S., Baragou S., N'djao J., Atta B., Ehlan E., Damorou F., Goeh-Akué E. (2014). Epidémiologie et étiologies des insuffisances cardiaques à Lomé. Pan African Medical Journal, 18:183.

7. Maliki Abdoulaye M, Harouna B, Harouna H, Tahirou I, Adehossi Omar E, Touré AI. (2018). Insuffisance cardiaque de l'adulte : Une étude sur 130 cas à l'hôpital au Service de cardiologie de l'hôpital national de Niamey au Niger. Jaccr Africa, 2(1) ,139 -145.

8. Aziouaz E. (2014). Le profil épidémiologique des cardiopathies ischémiques au centre hospitalier régional Al Fârâbî- Oujda (à propos 
de 185 cas).Thèse de Doctorat en médecine, Université Sidi Mohammed ben Abdellah, Faculté de médecine et de pharmacie de Fès, ,99 $\mathrm{p}$.

9. Dioum M, Mbaye A, Ngaide AA, leye M, Mingou JS, Gaye N D, Ndiaye M, Cisse AF, Ka MM, Faye MM, Kouame I, Dieng M, Aw F, Sarr SA, Bodian m, Diagne D, Kane M, Diack B, Kane A. (2017). Les thromboses veineuses des membres : aspects epidemiologiques, diagnostiques, therapeutiques et evolutifs : etude retrospective sur une période de 09 ans à propos de 148 cas colliges au service de cardiologie de l'hôpital général de grand Yoff de Dakar. Rev. Cames Santé, 5(1) ,79-82.

10. Kouakou N YN., Traore F., Tano M., Kramoh KE., Kakou JB A, Konin C., Kakou MG. (2015).Aspects épidémiologiques des accidents vasculaires cérébraux (AVC) aux urgences de l'institut de cardiologie d'Abidjan (ICA). Pan African Medical Journal, 21:160.

11. Machihude P, Afassinou YM, GoehAkue E, Baragou S, Koutche K, Belo M, Kumako V. (2015). Autres facteurs concourant aux accidents vasculaires cérébraux chez l'hypertendu traité au CHU Sylvanus Olympio de Lomé. Cames Santé, 3(1) ,89-93. 